\title{
¿Qué aporta la escucha al capital social? Lecciones de un proceso de aprendizaje de líderes públicos
}

\author{
What does Listening Contribute to Social Capital?: Lessons from a Learning \\ Process for Public Leaders
}

Xabier Barandiaran, Anne Murphy y María José Canel*

Resumen: Los estudios de administración pública afirman que el capital social es un recurso de valor intangible en la gestión pública y, como tal, da acceso al desarrollo y fortalece la democracia. Pero poco se sabe sobre cómo crear, gestionar y evaluar este recurso. Esta investigación trata de contribuir a la práctica del capital social poniéndolo en relación con la escucha activa. Apoyada en el análisis sobre un proceso de aprendizaje emprendido por un equipo de gobierno, la investigación evidencia que cuando se reflexiona colaborativamente en un nivel micro sobre interacciones reales con los ciudadanos, los líderes públicos estrechan la relación con la organización y con la sociedad, explicitan el valor de la misma y, con ello, se predisponen a identificar y articular el capital social, así como a trabajar más a favor del mismo. El artículo apunta algunas implicaciones para la gestión pública.

Palabras clave: capital social, escucha, aprendizaje activo, liderazgo público, gobernanza.

Abstract: Public administration studies claim that social capital is an intangible asset in public management, and that, as such, it provides access to development as also strengthens democracy. Yet little is known about how to create, manage and assess this resource. This

*Xabier Barandiaran es profesor de comunicación institucional y corporativa, Universidad de DeustoCampus de San Sebastián, Facultad de Ciencias Sociales y Humanas, Edificio Padre Arrupe-2º planta, despacho 210, Mundaiz, 50, 20012 Donostia-San Sebastián, España. Tel: 34943 326600, ext. 5665. Correo-e: xabier.barandiaran@deusto.es. ORCID: 0000-0002-7557-9331. Anne Murphy es consultora en organización y candidata de doctorado en lingüística y liderazgo, Department of Linguistics and English Language, Lancaster University, Lancaster, LA1 4YW, Reino Unido. Tel: +44 (0)1524 65201. Correo-e: a.murphy2@lancaster.ac.uk. ORCID: 0000-0002-0980-8183. María José Canel es catedrática de comunicación política y del sector público, Universidad Complutense de Madrid, Facultad de Ciencias de la Información, Avenida Complutense s/n, Madrid 28040, España. Tel: 34-91-3942167. Correo-e: mjosecanel@ucm.es. orcid: 0000-0002-5048-124X.

Artículo recibido el 7 de mayo de 2020 y aceptado para su publicación el 4 de marzo de 2021.

http://dx.doi.org/10.29265/gypp.v31i1.1011 
research attempts to contribute to the practice of social capital by linking it with active listening. Supported by an analysis on a learning process undertaken by a governmental body, this research shows that, when public leaders collaboratively reflect at a micro level about their real interaction with citizens, they close relationships both with the organization and with society, make it more explicit the value of the latter, and hence, increase their predisposition to identify and articulate social capital, as also to foster it. This research suggests some implications for public management.

Keywords: social capital, listening, action learning, public leadership, governance.

\section{INTRODUCCIÓN}

- 1 "capital social” es un concepto largamente acuñado en la investigación Cacadémica (Putnam, 1993, 2000; Fukuyama, 1995; por citar por el momento solo algunas referencias) que adquiere todavía mayor valor en contextos actuales en los que los ciudadanos de diversas democracias han incrementado su desinterés por la agenda pública, al hacerse más críticos con las instituciones y distanciarse de los asuntos públicos (Whiteley et al., 2016). Son contextos en los que, por una conceptualización excesivamente fragmentada del hecho político, es más difícil configurar escenarios compartidos por líderes políticos y ciudadanos (Putnam, 2010).

La necesidad de estos escenarios compartidos se ha hecho todavía más acuciante con la crisis mundial ocasionada por el covid-19, en la que se ha puesto a prueba la capacidad de los líderes públicos de todos los países de aunar a las sociedades en torno al diagnóstico del problema y a las soluciones. Parece todavía más necesario que antes trabajar para incrementar el capital social.

Sin embargo, al tratarse de algo intangible, resulta complejo identificar qué políticas gubernamentales pueden ayudar a incrementar este tipo de capital. De hecho, la mayor parte de la literatura se enfoca más en el análisis del concepto o en consideraciones normativas sobre su contribución a la democracia. Escasean los trabajos más orientados al desarrollo de las relaciones en las que se apoya este recurso. De acuerdo con Bolívar y Flores (2011: 29), "los resultados empíricos dejan pendiente una pregunta crucial: ¿Cómo producir capital social?".

La presente investigación trata de contribuir en esta dirección. Ha sido posible, primero, porque se centra en lo que hace un equipo de gobierno involucrado en un programa de gobernanza colaborativa (es decir, que persigue la construcción de escenarios compartidos por gestores públicos y ciudadanos) y, por lo tanto, porque se atiene a la situación real en la que los líderes públicos llevan a cabo su desempeńo; y, segundo, porque para implementar mejor el programa, el gobier- 
no tomó la decisión de llevar a cabo un proceso de aprendizaje sobre la escucha a la sociedad cuyo enfoque implicaba explicitar lo aprendido. Experiencia real de construcción de escenarios compartidos y explicitación de lo aprendido se presentaban como características propicias para explorar el carácter relacional e intangible del capital social.

El gobierno objeto de análisis emprendió el proceso de aprendizaje apoyado en la asunción de que la escucha (entre los líderes públicos y de los líderes públicos hacia la sociedad) puede ser buena para estrechar las relaciones que son necesarias para implicar a los ciudadanos en la gestión pública. En el detallado análisis que realiza sobre el estado de la escucha organizacional, Macnamara $(2015,2016,2017)$ afirma que las organizaciones públicas practican la escucha a través de prácticas como la consulta pública o la gestión de quejas. Pero este y otros autores consideran que el objetivo de mejorar los procesos de escucha a la sociedad pasa por hacer, primero, que la propia organización escuche más. Por ejemplo, Suárez et al. (2018) destacan la importancia de crear un nuevo escenario en la gestión pública que promueva el intercambio de conocimiento, la construcción de diagnósticos compartidos y la consolidación de propuestas de soluciones y trabajo colectivo, y en el que se involucran las instituciones públicas (Suárez Rincón et al., 2018). Prieto y Fabelo (2009: 54) hablan de reorientar las acciones de los líderes hacia la creación de tejidos sociales capaces de significar un empoderamiento real de la sociedad.

Esta investigación toma entonces como objeto de análisis un proceso de aprendizaje activo desarrollado al interior de un equipo de gobierno provincial (el de la Diputación Foral de Gipuzkoa en el País Vasco) en el contexto de un programa gubernamental que tenía por objeto implicar a los ciudadanos en las decisiones de gestión pública. El objetivo es explorar si la escucha aporta al capital social, y más concretamente, determinar si se puede asociar la reflexión de líderes públicos en torno al aprendizaje de escucha a una mayor predisposición por parte de estos a la construcción de capital social, con el fin, en último término, de identificar acciones beneficiosas para una sociedad.

La estructura del artículo es la siguiente. Primero se revisa el marco teórico con el fin de explorar los conceptos que se quieren poner en relación, a saber, la escucha y el capital social, y formular la pregunta de investigación. A continuación se explica la metodología. Tras la exposición de resultados se presentan las conclusiones, incluyendo unas consideraciones operativas sobre la gestión del capital social en las organizaciones públicas. 


\section{MARCO TEÓRICO}

\section{La escucha en el liderazgo público}

La importancia de la escucha para la calidad de la democracia es una consideración normativa altamente presente en la literatura en general, y en los estudios de liderazgo en particular (Stivers, 1994; Ospina, 2016; Crosby y Bryson, 2017). Los autores consideran que el diálogo que lleva asociado la escucha ayuda a configurar una realidad compartida, un espacio público común (Fernández et al., 2018). Se argumenta que la capacidad de dar respuesta a las demandas de la sociedad comienza por la escucha, por lo que los líderes públicos no pueden obviar esta tarea (Needham, 2001; Johnson, 2007; Macnamara, 2015, 2017; Canel, 2018).

Esta relevancia de la escucha queda bien expresada en la afirmación de Bellah et al. (1991: 254) de que "democracia significa prestar atención". Para poder efectivamente prestar atención, es preciso que los líderes públicos desarrollen sus propias habilidades de escucha con el fin de estar en sintonía con la sociedad y así brindarle un mejor servicio. Con mejores habilidades de escucha se tienen en cuenta voces olvidadas, se entabla una comunicación recíproca, se promueve la rendición de cuentas, se comprenden mejor las situaciones complejas y se adoptan los enfoques interactivos propios de los procesos participativos (Korten, 1981; Stivers, 1994; Borner y Zerfass, 2018; Carvalho et al., 2019; Ercan et al., 2019). La escucha, en fin, implica apertura, respeto por la diferencia y reflexividad (Stivers, 1994; Simmons, 2011).

La literatura establece también que la escucha es un elemento central en la generación de confianza. Respalda las reivindicaciones de legitimidad de los gobiernos democráticos (Dobson, 2012), y su contrario, la no-escucha, puede hacer perder cualquier confianza adquirida (Forester, 1989; Beshi y Kaur, 2019). En definitiva, una mejor escucha confiere legitimidad a las decisiones (Dobson, 2012), idea que está relacionada con la consideración de algunos autores de que la cualificación democrática de una medida puede tener que ver más con el proceso con el que se aprobó que con el contenido de la misma (Richards, 2008; Canel et al., 2017).

Los estudios de liderazgo teorizan sobre la escucha como dimensión esencial para un liderazgo efectivo (Southard y Wolvin, 2009). Heifetz expande el modelo del líder que escucha: "La buena escucha — dice — se alimenta de la curiosidad y la empatía. Es difícil ser un gran oyente si no te interesan los demás" (Heifetz, y Linsky, 2017: 65). Sin embargo, a pesar de reconocer el papel crucial que desempeña la escucha en el liderazgo, es frecuente encontrar líderes públicos que realizan solo comunicación de mensajes (Southard y Wolvin, 2009) en una rela- 
ción unidireccional (Macnamara, 2015). Parece mostrarse que queda camino por recorrer en el desafío de apoyarse en la escucha para configurar espacios y escenarios compartidos con la sociedad a la que se sirve y ejercer así mejor el liderazgo público.

\section{El capital social, un recurso intangible de la gestión pública}

Dicho ahora de manera algo simple, el capital social tiene que ver con el valor que pueden alcanzar las relaciones. La teoría del capital social examina cómo las relaciones sociales, una vez que se forman, albergan la capacidad de beneficiar no solo a los individuos sino también a las organizaciones, llevándolas más allá de su contexto originario: el capital social facilita que la gente colabore, socialice, establezca comunidades y viva conjuntamente en armonía (Coleman, 1990; Lin, 2001; Portes, 1998).

Siguiendo a uno de los autores paradigmáticos sobre el concepto, el capital social hace referencia a las conexiones entre los individuos, las redes sociales y las normas de reciprocidad y confianza que de esas relaciones surgen (Putnam, 2000: 19). Se puede decir que entidades o países con alto capital social son, también, los que cuentan con nutridas redes en las que cada parte sabe qué puede esperar de y hacer con el resto.

En la exploración de las visiones que hay en el estudio del capital social, Gutiérrez (2008) destaca las aportaciones de Coleman (1988) y de Bourdieu (1983), quienes se acogen a una perspectiva estructural, es decir, la que relaciona las condiciones objetivas externas (históricamente situadas) con aquellos elementos que permiten ubicar a los agentes e instituciones en el espacio social. Se otorga una mayor relevancia al análisis de las estructuras formales e informales que dan fundamento a las interacciones.

Desde esta perspectiva, para Coleman (1988) el capital social puede tomar muchas formas, entre las que están las obligaciones que hay dentro de un grupo, las normas y las sanciones. Para Bourdieu (1983), el concepto de capital social subraya las relaciones sociales que incrementan la capacidad de un actor de promover sus intereses. De nuevo aparece la idea de que el capital social permite al individuo específico ir más allá de sí mismo/a en la medida en que le facilita la colaboración y el compromiso, así como el flujo de información; mediante un sentido de pertenencia y obligación social, puede ejercer influencia sobre los demás (Saffer, 2016).

Uno de los puntos centrales a efectos de la presente investigación es que hablar de capital social implica entender que las relaciones son fuente de desarrollo. 
Autores como Bourdieu (1983), Coleman (1988, 1990), Fukuyama (1995), Putnam (2000) y otros científicos sociales, afirman que el nivel de confianza interpersonal, el compromiso cívico y la capacidad organizativa en una comunidad - todos ellos componentes del capital social— son factores fundamentales para el desarrollo.

De hecho, algunos trabajos conceptualizan el capital social como un recurso intangible de las organizaciones (Canel y Luoma-aho, 2019), apoyándose en la idea de que las relaciones permiten acceder a bienes y recursos que de otra forma serían inalcanzables para los individuos (Coleman, 1988; Bourdieu, 1983). Recientes investigaciones en el ámbito de las relaciones públicas organizacionales argumentan que el capital social proporciona marcos de evaluación tanto del "valor intangible (por ejemplo, las relaciones, la reputación, la confianza) como del tangible (por ejemplo, el beneficio financiero) que proporcionan las actividades de relaciones públicas" (Dodd et al., 2015: 473).

Putnam (2000: 19) explicita esta consideración al afirmar que, mientras que el capital físico se refiere a los objetos físicos, y el capital humano a las propiedades de las personas individuales, el capital social tiene que ver con las conexiones que hay entre las personas. Ilustra esta idea afirmando que "una sociedad llena de individuos virtuosos pero aislados entre sí no sería una sociedad rica en capital social".

Como recurso intangible que es, el capital social da acceso a bienes y recursos tangibles. Puede generar valor en múltiples niveles, bien sea el individual, el grupal o el comunitario, por lo que es legítimo tomar el capital social como una característica agregada de las naciones. En este sentido, una amplia gama de estudios evidencia que sociedades ricas o densas en capital social disfrutan de un conjunto de beneficios comunes, tales como atenuar la pobreza y la exclusión social, facilitar el desarrollo económico, mejorar la búsqueda de empleo, influir en el rendimiento educativo, mejorar el bienestar personal y la salud, facilitar la gobernanza y la participación ciudadana y disminuir la delincuencia (Fukuyama, 1995; Lin, 2001; Hyyppä y Mäki; 2003; Putnam et al., 2003; Luoma-aho et al., 2012). De ahí que diversas agencias internacionales hayan adoptado el capital social como un instrumento para mejorar las condiciones de vida de las personas (Ordóñez y Ruiz Ochoa, 2015).

En definitiva, el capital social representa la idea de que las relaciones sociales pueden facilitar la producción de bienes económicos o no económicos, ya sean tangibles o intangibles, y de ahí la dificultad para llevar a cabo programas de identificación, gestión y evaluación del capital social: su construcción pasa por 
procesos que son de largo plazo, como los procesos de escucha, cuestión que se aborda en la siguiente sección.

\section{La construcción de capital social a través de la escucha activa}

La literatura tiende a asociar la escucha al capital social. Uno de los argumentos que se esgrime es precisamente el carácter relacional del capital social, por cuanto no hay relación si no hay interacción. Afirma Barreiro que el capital social se diferencia de otros factores de desarrollo en que es el único que es relacional, se encuentra en la estructura de las relaciones (Barreiro, 2000). La construcción del capital social pasa por que las personas o las organizaciones interactúan, y al entender de Gallicchio (2004), esta continuada interacción social es constituyente del capital social.

Como se ha afirmado en la sección anterior, el capital social nace de relaciones que generan orgullo de pertenencia y visiones compartidas de problemas y soluciones, y la escucha es necesaria para que tal visión compartida se produzca pues, como considera Plati (2005), la escucha puede poner a una sociedad frente a problemas emergentes y facilitarle dar con una respuesta común y proactiva.

Otro argumento para conectar el capital social con la escucha es el que afirma una relación positiva entre capital social y democracia. La teoría democrática y las recientes iniciativas de política internacional establecen esta relación (Yang y Taylor, 2013; Åberg y Sandberg, 2017) apoyándose en que, porque el capital social contribuye a la creación de espacios críticos — en los que los distintos pareceres tienen cabida-, puede no solo mejorar la calidad de democracias existentes sino también introducir democracia en contextos no democráticos.

Con apoyo en las aportaciones de diferentes autores (Hazelton y Kenna, 2000: 83; Luoma-aho, 2013: 282; Canel y Luoma-aho, 2019: 253; Canel, 2018; Johnston y Lane, 2018; Ramírez de la Cruz, 2019; Piqueiras et al., 2020), se puede afirmar, además, que varias de las acciones que ayudan a construir capital social requieren de procesos de comunicación en los que necesariamente hay escucha: el intercambio de información, la identificación de problemas así como de soluciones, el alineamiento de los empleados públicos con las metas organizacionales, la gestión del conflicto, y la confianza necesaria para asumir el riesgo que lleva consigo la innovación.

La literatura afirma también que el capital social proporciona valor a la sociedad mediante la confianza y la confiabilidad (Coleman, 1990; Fukuyama, 1995). La confiabilidad como forma de capital social incrementa "la capacidad de formar nuevas asociaciones" (Fukuyama, 1995: 27). Se puede entender entonces que las 
organizaciones públicas que cuentan con redes de stakeholders de confianza recíproca tienen un alto valor de capital social (Canel y Luoma-aho, 2019: 248).

La aportación de Putnam et al. (2003) es de gran utilidad para analizar y plantear la generación de capital social en la gestión pública. Considera este autor que es clave lograr que la gente se implique con los demás primero a escala menor, y estas experiencias de trabajar conjuntamente a pequeńa escala constituyen la base para generar confianza al conjunto de la sociedad, pues estos grupos actúan como bases de aprendizaje para la democracia (Putnam et al., 1993). La construcción de esta interacción que está en la base del capital social requiere entonces de procesos de comunicación y escucha que se tendrán que hacer primero en grupos pequeños para redundar luego a la sociedad en general.

Es preciso realizar un último apunte. Para construir capital social hay que movilizarlo, explicitarlo, pues es cuando se explicita la potencialidad de las relaciones (por ejemplo, una confianza que quizá estaba latente), cuando se incrementa el valor que las mismas entrañan. Por eso, a diferencia de otros recursos intangibles (como la reputación), el capital social no se construye intencionalmente como resultado de un plan, sino que deriva de procesos que establecen relaciones sociales de largo plazo, que generan experiencias positivas en los implicados, y a través de los cuales se explicita la existencia de las mismas (Canel y Luoma-aho, 2019).

La revisión de la literatura realizada hasta aquí lleva a afirmar las siguientes características del capital social: a) tiene que ver con relaciones que se establecen cuando las personas y organizaciones se implican para definir conjuntamente los problemas y las soluciones a los mismos; $b$ ) se establece y desarrolla yendo de un nivel micro (primero en un grupo pequeño) a un nivel macro; es decir, las relaciones se extienden y, con ello, incrementan el nivel de capital y el alcance de sus "propietarios"; c) es de carácter intangible y, por lo tanto, los procesos de explicitación colaborativa pueden contribuir a su identificación y refuerzo. Esta revisión de la literatura permitió formular la pregunta de investigación en los siguientes términos:

PI: ¿Qué aporta al capital social la reflexión colaborativa de lideres públicos sobre su aprendizaje de escucha?

\section{LA ESCUCHA ACTIVA PARA EL DESARROLLO DE GOBERNANZA COLABORATIVA EN ETORKIZUNA ERAIKIZ}

La presente investigación toma como objeto la reflexión de un equipo de gobierno que decide aprender a escuchar para implicar a la sociedad en la gestión pública. 
Se trata del gobierno de la provincia de Gipuzkoa, la Diputación Foral de Gipuzkoa, autoridad pública dentro de la Comunidad Autónoma Vasca, capital San Sebastián. Hay que decir que Gipuzkoa es un territorio en el que hay cierta cultura de tejido social para el abordaje de soluciones a los problemas públicos, y que el propio Robert Putnam tuvo ocasión de conocer en una visita que realizó en 2010, cuando el programa gubernamental que aquí se analiza estaba todavía en una fase anterior. Considerando las especiales circunstancias del momento (el País Vasco padeció hasta el año 2010 el azote del terrorismo por parte de ETA), Putnam apreció la potencialidad de la región para ver en la situación conflictiva ocasión de construir capital social y hacer de este palanca para la prosperidad social y económica (Putnam, 2010).

En el año 2015 la Diputación Foral de Gipuzkoa inició la construcción de un sistema de gobernanza abierta y colaborativa, que consiste en una nueva dinámica de cooperación junto con otros muchos actores del territorio, no solo para elaborar la agenda pública compartiendo sus contenidos, objetivos y prioridades, sino también para coparticipar con la sociedad en la implementación de políticas públicas. Este objetivo se materializó en Etorkizuna Eraikiz ("Construyendo el futuro" es la traducción de la lengua vasca), que incluye proyectos en áreas distintas como la sanitaria, cultural, igualdad, inmigración, promoción del territorio, etcétera.

Etorkizuna Eraikiz pone la escucha, la reflexión y la experimentación en el centro con el fin de redirigir las políticas públicas y promover la innovación en el sistema de gobernanza institucional, fortaleciéndolo, dinamizándolo y adaptándolo a la sociedad a la que sirve. A través de espacios de escucha, deliberación y decisión, no regidos por la jerarquía sino por las relaciones horizontales y en los que el gobierno interactúa con la sociedad civil (instituciones, empresas, organizaciones no gubernamentales, universidades, asociaciones ciudadanas, etc.), se coopera para construir conjuntamente la agenda, y diseńar y experimentar las políticas públicas. Fruto de esta colaboración, reflexión, intercambio de pareceres y diálogo, se genera conocimiento y aprendizaje con el que se identifican retos y oportunidades relevantes para el futuro (Diputación Foral de Gipuzkoa, 2019).

Esta manera de entender la gobernanza implica importantes cambios en las estructuras y los procesos. Por ejemplo, se estableció una nueva manera de elaborar el prespuesto, que ya no es departamental sino por proyectos; o se creó una nueva unidad (Dirección General de Gestión Estratégica) para llevar a cabo una gestión más transversal e interdepartamental. Todos estos cambios tienen importantes repercusiones en la manera en que los diferentes actores interactúan, y 
por ello la escucha ha emergido como reto importante durante los cinco años de avance en la implementación de este modelo de gobernanza. Para hacer frente a este desafío y desarrollar aprendizajes individuales y organizacionales en torno a la escucha de los líderes públicos, la propia diputación se planteó llevar a cabo una intervención que tuvo por título "Aprender escuchando a la sociedad", con el objetivo de construir un espacio de aprendizaje con reflexión sobre la escucha interna (entre políticos y funcionarios). Este artículo analiza este proceso con el fin de explorar si el aprendizaje de la escucha puede aportar a la construcción y al fortalecimiento del capital social.

\section{METODOLOGÍA}

El diseño metodológico de la presente investigación está informado por el hecho de que el objeto de análisis es la práctica real de un equipo de gobierno cuando se dispone a aprender a escuchar a la sociedad para implementar mejor un programa de gobernanza colaborativa.

El proceso de aprendizaje analizado siguió el enfoque denominado Action Learning (Pedler, 2011; Revans, 2011). Este método de aprendizaje experimental se fundamenta en el principio de que los participantes aprendan de las acciones que implementan con el objetivo de progresar en la resolución de problemas.

De acuerdo con Revans (1998), este proceso de aprendizaje tiene dos componentes. El primero de ellos consiste en un aprendizaje programado en el que un conjunto de participantes proporciona información sobre problemas organizacionales considerados enrevesados o intratables con el fin de identificar soluciones por medio de la acción. Los participantes formulan preguntas para ayudarse mutuamente a explorar las situaciones que enfrentan. El segundo componente es el de la reflexión sobre la acción: tras el análisis del problema, cada participante se compromete con una acción para abordarlo, acción sobre la que reportará en la siguiente reunión para, de nuevo con la ayuda del grupo, reflexionar sobre lo aprendido.

Por lo tanto, el despliegue de este proceso al interior de la Diputación Foral de Gipuzkoa se inició con la configuración de un grupo de action learning (AL) conformado por los doce responsables políticos (la mayor parte funcionarios electos y dos funcionarios públicos de carrera) que componen el equipo de gobierno provincial y, por lo tanto, con la máxima responsabilidad deliberativa y de decisión. El anexo 1 detalla las características de los miembros del grupo.

El proceso se extendió durante seis meses, con la celebración de un total de cinco sesiones a lo largo del año 2019 (sesión 1: 13 de julio; sesión 2: 7 de sep- 
tiembre; sesión 3: 19 de octubre; sesión 4: 30 de noviembre; sesión 5: 10 de diciembre) de aproximadamente seis horas cada una, facilitadas por dos especialistas. Como el enfoque de aprendizaje requiere la acción —en cada sesión se reflexiona sobre las acciones implementadas-, esta intervención llevó consigo, por un lado, la aplicación de acciones para interactuar mejor, como por ejemplo experimentar nuevas formas de organizar los actos públicos o desarrollar encuentros con nuevos sectores de la población; por otro, y en consecuencia, supuso una elevada interacción real de los miembros del equipo de gobierno con las organizaciones participantes en Etorkizuna Eraikiz (empresas, asociaciones ciudadanas, universidades, o usuarios de los servicios públicos).

Pero action learning no fue la metodología empleada en la presente investigación sino la vía a través de la cual generar los datos para la misma. Al final de cada sesión, y fuera ya del requerimiento de confidencialidad propio de ese enfoque, se administró a los participantes (la muestra es la totalidad del equipo de gobierno, que participó en todas las reuniones) un cuestionario con el fin de propiciar una reflexión que explicitara el aprendizaje. Los participantes entregaron las respuestas por escrito y a continuación se les pidió que discutieran en grupo sus consideraciones. Por lo tanto, los datos de la presente investigación no capturan la interacción de los líderes públicos con las distintas organizaciones (que, por cierto, fue muy elevada dadas las características del enfoque de aprendizaje), sino la reflexión de los mismos sobre esa interacción. La base de datos para la presente investigación está compuesta de las respuestas de los participantes (se registraron un total de 643 unidades de análisis), así como de las notas tomadas por los autores sobre las discusiones en grupo.

El cuadro 1 proporciona, por un lado, información sobre el contexto en el que se produce la interacción entre los miembros del grupo (el tipo de preguntas a las que se expusieron entre ellos) y, por otro, las preguntas de reflexión posterior que propiciaron los datos en los que se apoya la presente investigación.

Los datos fueron analizados cualitativamente, identificando los temas recurrentes; además, se expusieron de forma evolutiva, es decir, se extrajo de cada sesión la idea esencial, de manera que el despliegue cronológico pudiera aportar conocimiento sobre una posible evolución en el proceso de aprendizaje.

En una investigación precedente encontramos que la implementación del action learning generó en el equipo de gobierno un "espacio de criticidad" (en el que se hace posible afrontar las inercias identificando y describiendo los obstáculos para el cambio organizacional) del que derivó un aprendizaje de escucha entre los líderes y, en consecuencia, hacia la sociedad (Murphy et al., 2019). En esta inves- 
CUADRO 1. Ejemplos de preguntas para la exploración de los problemas en el action learning y para la reflexión posterior

Preguntas formuladas por los participantes en las sesiones de action learning

Preguntas de reflexión posterior

- ¿Qué quiere decir "escuchar a la sociedad"?

- ¿Cómo se puede llegar de manera más efectiva a los

¿Qué has aprendido hoy sobre: ciudadanos?

- el proceso de reflexión y el grupo?

¿Cómo se puede involucrar más a los funcionarios

$-\mathrm{ti}$ ? públicos?

- los problemas de la gestión pública?

- la Diputación Foral?

- ¿Cómo se puede aumentar la capacidad de

-Etorkizuna Eraikiz? transformación de las políticas públicas en cada

- el proceso de escucha? departamento/área de la diputación?

- ¿Qué piensan los ciudadanos acerca de este programa de políticas públicas Etorkizuna Eraikiz?

- ¿Qué es importante para los ciudadanos?

- ¿Se está abierto a escuchar las críticas de los ciudadanos?

Fuente: Elaboración propia.

tigación atendemos específicamente a si en ese espacio se pudieron dar las condiciones para que quienes participaron en él, se predispusieran mejor a un capital que, como el social, es relacional e intangible. Para dar respuesta a la pregunta de investigación, se buscaron específicamente aquellos temas recurrentes en la reflexión sobre lo aprendido que pudieran contener señales sobre las características propias del capital social que se han extraído de la revisión de la literatura expuesta en la primera parte de este artículo.

\section{RESULTADOS Y DISCUSIÓN}

Esta sección expone y discute los principales resultados obtenidos, que se presentan siguiendo cada uno de los aspectos de aprendizaje planteados en la reflexión, y que responden a las cuestiones ya mostradas en el cuadro 1 . Se despliegan los datos cronológicamente para poder identificar la evolución en el proceso. Para consignar tal evolución, se ha extraído de cada sesión la idea más recurrente en las respuestas que los participantes dieron a cada pregunta específica. 


\section{Aspecto 1: Aprendizaje sobre el proceso de aprendizaje: \\ La experiencia de la explicitación colaborativa}

El cuadro 2 recoge los datos sobre las respuestas proporcionadas a la pregunta sobre el proceso de reflexión desarrollado en el aprendizaje.

CUADRO 2. Aspecto 1: Evolución del aprendizaje sobre el proceso de aprendizaje

¿Qué has aprendido hoy sobre el proceso de aprendizaje?

Sesión AL 1 La importancia de formular las preguntas adecuadas.

Sesión AL 2 Que hay distintas visiones sobre los problemas.

Sesión AL 3 Que compartir es clave para que se pueda desarrollar aprendizaje.

Sesión AL 4 La importancia de desarrollar el espíritu crítico y la escucha activa entre los líderes públicos para generar cohesión grupal con la que se explicita el aprendizaje de forma colaborativa.

Fuente: Elaboración propia.

Los datos revelan características propias de la metodología de aprendizaje empleada: para aprender es necesario partir de que no se sabe, y ayudar a avanzar planteando buenas preguntas (Revans, 1998). Así, al inicio del proceso, los participantes se hacen conscientes de la importancia de aprender a formular las preguntas adecuadas y documentar la visión compartida por cada uno frente a las cuestiones expuestas. Se siente que se aprende con y de los demás, para lo que hay que "prestar atención, fortalecer la capacidad de escucha y activar la interacción”, según expresó un participante.

El avance de las sesiones evidenció una mayor conciencia de la necesidad de reservar tiempo al proceso de aprendizaje, tanto individual como colectivo, así como de la importancia de adoptar una actitud abierta al aprendizaje. Es un contenido recurrente en las respuestas de los participantes la necesidad de mantener estos procesos en el tiempo y de dotar de herramientas a los líderes públicos en los ámbitos personal, colectivo y organizacional.

En la última sesión del proceso se evidencia que los participantes han fortalecido el espíritu crítico y la escucha activa entre pares, generando confianza y cohesión: "Ha sido un grupo de aprendizaje diverso, con distintas visiones, muy plural, pero con un importante nivel de autoreconocimiento como grupo, y esto ha generado un importante nivel de cohesión grupal”. Otra persona 
afirma: "Soy más consciente ahora de que soy más capaz de actuar de otra manera tras este proceso. Me ha servido para cohesionarme de un modo distinto con el grupo".

Parece ponerse de manifiesto que la reflexión sobre el aprendizaje de escucha incrementó en el equipo de gobierno no solo la conciencia de existencia de unas relaciones más estrechas dentro del grupo, sino la explicitación del valor de las mismas ("Lo importante es la relación que se establece" dice, por ejemplo, una participante). Emerge como idea recurrente que el hecho de poder compartir lo aprendido con los demás ayuda a hacerse más consciente de realidades que, de otra manera, permanecerían latentes y ocultas, en este caso, unas relaciones más sólidas.

\section{Aspecto 2: Aprendizaje individual de los líderes públicos: La conciencia de espacios compartidos}

En las respuestas proporcionadas a la pregunta sobre el aprendizaje individual, los datos muestran la evolución hacia una mayor conciencia de la necesidad de intercambiar comunicación para pasar de visiones individuales de los problemas a definiciones realizadas de forma conjunta. En el orden individual existe una dificultad para dejar de lado las dinámicas cotidianas de la organización y compartir problemas de manera colectiva con el fin de buscar soluciones que impliquen a otras áreas de la organización o a la propia sociedad. En palabras de los líderes públicos que participaron en el proceso "se debería dar mayor relevancia a priorizar lo importante/estratégico sobre lo urgente. Tenemos que dedicar más tiempo de nuestro día a día a elementos que definimos como estratégicos, como por ejemplo el contacto con la sociedad".

A medida que el proceso de aprendizaje avanza en el tiempo, se refleja una evolución en la percepción de los problemas - muy individualizados al principio- así como en la racionalización de los mismos a través de la escucha de otros pares, aumentando la empatía y la consideración de que en la gestión pública, los problemas son más compartidos de lo que los participantes imaginaban al comienzo: "He aprendido que mi problema tiene que ver con la mayoría de los problemas de mis compañeros/as". El cuadro 3 recoge la evolución del aprendizaje individual.

Con el desarrollo del proceso se va explicitando el valor de la escucha para pensar de forma colectiva, así como para construir espacios compartidos en los que desarrollar visiones comunes sobre las situaciones: "Deberíamos fortalecer el convencimiento de la importancia de la escucha y de los procesos de construcción 
CUADRO 3. Aspecto 2: Evolución del aprendizaje individual en los líderes públicos

\begin{tabular}{ll}
\hline ¿Qué has aprendido hoy sobre ti? \\
\hline Sesión al 1 & $\begin{array}{l}\text { La dificultad para dejar de lado las dinámicas cotidianas y compartir problemas de } \\
\text { manera colectiva. }\end{array}$ \\
Sesión al 2 & $\begin{array}{l}\text { El valor de la escucha en el grupo para dimensionar el problema individual y } \\
\text { racionalizarlo en relación con el grupo. }\end{array}$ \\
Sesión al 3 & $\begin{array}{l}\text { Que se comparten visiones sobre los problemas, las soluciones y los retos de futuro, no } \\
\text { solo con el grupo, sino también con las organizaciones sociales y los ciudadanos. }\end{array}$ \\
Sesión al 4 & $\begin{array}{l}\text { El valor de la escucha para aportar a los procesos de pensamiento colectivo con los } \\
\text { que definir colaborativamente problemas y soluciones. }\end{array}$
\end{tabular}

Fuente: Elaboración propia.

y aprendizaje colectivo". La escucha fue reconocida como una oportunidad para que todas las partes interesadas puedan expresar sus necesidades, creencias y percepciones en relación con un problema, tema o proyecto.

La evolución de las opiniones sobre el aprendizaje individual refleja también que la creación de espacios para la escucha y la colaboración grupal refuerzan la evaluación de las situaciones antes de plantear soluciones. Mantener la atención hacia el otro/a añade nuevas visiones o matices diversos frente a los temas. En definitiva, el proceso de aprendizaje y escucha ha facilitado visiones compartidas de los problemas y de las soluciones, algo que, como se recoge en la revisión de la literatura, está en la base de la creación del capital social.

\section{Aspecto 3: Aprendizaje sobre los problemas de la gestión pública: Del nivel micro al nivel macro}

Una tercera pregunta de reflexión que se planteó a los participantes tras la sesión de action learning fue: ¿Qué has aprendido hoy sobre los problemas/proyectos?

El cuadro 4 recoge la evolución del aprendizaje en este aspecto.

$\mathrm{Al}$ comienzo del proceso de aprendizaje los participantes constatan que se desconfía de la capacidad de la sociedad de responder e implicarse en la gobernanza colaborativa que se quiere llevar a cabo: "La sociedad está alejada de la política"; "la sociedad es muy exigente" "la sociedad no quiere participar". Pero se constata desconfianza también en los líderes públicos hacia su propia capacidad de llegar a la sociedad: "No sabemos realmente hacia dónde va la sociedad, 
CUADRO 4. Evolución del aprendizaje de los líderes públicos sobre los problemas de la gestión pública

¿Qué has aprendido hoy sobre los problemas de la gestión pública?

Sesión AL 1

Se constata una situación de partida en la que los líderes públicos juzgan que la sociedad desconfía de los políticos y la política.

Sesión AL 2 Que hay también desconfianza en los gestores públicos sobre su propia capacidad de llegar a la sociedad para abordar conjuntamente la gestión pública.

Sesión AL 3

Que para llegar a la sociedad, la gestión de la escucha tiene que asegurarse en un nivel micro la autenticidad y honestidad.

Sesión AL 4

Que cuando se escucha, se conoce mejor a la sociedad y, en consecuencia, se puede llegar a confiar más en ella.

Fuente: Elaboración propia.

o mejor dicho, dónde encontrarnos con ella". Incluso uno llegó a escribir: "No llegamos a la sociedad, no conectamos y no acompañamos en la evolución. ¿Qué hacemos?"

A medida que el proceso de aprendizaje avanza se hace recurrente la consideración de que la escucha tiene que ser auténtica. Véanse los siguientes extractos proporcionados por distintos participantes: "Si existe un ejercicio sincero, continuo y bien estructurado de escucha por parte de los líderes públicos, se puede fomentar un cambio en la mentalidad ciudadana hacia lo público"; "el acercamiento tiene que ser sincero y no cosmético"; "tenemos que transcender a la cosmética y al marketing". Parece como si la reflexión sobre la continuada interacción con los actores de los proyectos hubiera ido acompañada de un desafío mutuo en el grupo sobre la autenticidad de los intentos de escucha.

Los datos revelan que los participantes se han hecho conscientes de la necesidad de producir primero un cambio individual en ellos para de verdad escuchar y encontrarse con la sociedad; y de que cuando tal se produce, se descubre una sociedad que está más preparada, interesada y deseosa de participar en la gobernanza de lo que inicialmente se esperaba. "La sociedad es más abierta y tolerante y menos prejuiciosa de lo que esperaba"; "la sociedad tiene un gran potencial”, son afirmaciones de participantes en la última sesión.

La desconfianza inicial del líder tanto en la propia capacidad como en la de la sociedad a la que quiere implicar parece haberse tornado en confianza primero en 
un nivel micro — entre los participantes del grupo — para luego escalar a un nivel macro - el del conjunto de la sociedad-. Es preciso recordar aquí que los datos no están registrando la confianza real de los miembros del gobierno hacia la sociedad, sino el reporte que ellos hacen sobre lo que han aprendido al interactuar con la sociedad. Y parece ser el caso que, al reflexionar sobre la interacción mantenida con las organizaciones participantes en los proyectos de Etorkizuna Eraikiz, estos líderes manifiestan sentir una mayor confianza tanto hacia el interior del grupo como hacia la sociedad a la que quieren implicar, lo cual ofrece una base que respalda la idea de que esa predisposición a consolidar relaciones se ha generado primero a una escala menor (con planteamientos concretos y personales micro), para pasar después a un nivel más macro (con debates más amplios y globales sobre retos futuros que son importantes para el conjunto del territorio).

\section{Aspecto 4: Aprendizaje sobre la propia organización y su concepto de gobernanza: El reconocimiento de la propia capacidad tractora de toda la sociedad}

Se planteó también a los participantes la siguiente pregunta: ¿Qué has aprendido hoy sobre la diputación y su modelo de gobernanza? Los datos se recogen en el cuadro 5.

CUADRO 5. Aspecto 4: Evolución del aprendizaje sobre la propia organización y su concepto de gobernanza

¿Qué has aprendido hoy sobre la diputación y Etorkizuna Eriaikz

$\begin{array}{ll}\text { Sesión AL } 1 & \begin{array}{l}\text { La necesidad de establecer mecanismos que doten a la organización de agilidad y } \\ \text { flexibilidad. }\end{array} \\ \text { Sesión AL 2 } & \begin{array}{l}\text { Que hace falta adaptar la estructura organizacional al modelo de gobernanza abierto y } \\ \text { colaborativo. }\end{array} \\ \text { Sesión AL } 3 & \begin{array}{l}\text { La necesidad de consolidar esfuerzos para extender la dinámica del proyecto de } \\ \text { gobernanza al interior de la organización. }\end{array} \\ \text { Sesión AL4 } & \begin{array}{l}\text { Que la gobernanza colaborativa genera no solo cambio organizacional sino también } \\ \text { transformación social. }\end{array}\end{array}$

Fuente: Elaboración propia.

Los datos reflejan que el aprendizaje de los participantes evoluciona de una fase inicial, en la que se constatan deficiencias de la propia organización, a una 
fase final, en la que hay mayor confianza en la capacidad del modelo de gobernanza que se pretende desarrollar. Así, las respuestas de la primera sesión diagnostican una Diputación Foral que adolece de estructura antigua, diseñada en 1980. Según lo informado por los participantes: "La diputación es una organización profesional y madura pero necesita establecer mecanismos que le doten de agilidad y flexibilidad para adaptarse a los cambios que se generan en la actualidad y que vendrán en el futuro". Se observa la dificultad al interior de la organización para modernizar la propia estructura y dar el salto necesario.

A medida que el proceso de aprendizaje avanza, los participantes ven una organización que tiene más capacidad de impacto de lo que al principio consideraban: "Somos pequeños, invisibles, pero tenemos mucha capacidad de incidir en el futuro de la sociedad, debemos canalizar bien esta influencia”, es la afirmación de un participante. El aprendizaje incrementa la conciencia de que la propia estructura organizacional debe y puede adaptarse al modelo de gobernanza abierto y colaborativo que se persigue; pero la conciencia, además, de que el modelo de gobernanza que escucha a la sociedad es un mecanismo de transformación no solo organizacional ("Etorkizuna Eraikiz se ha convertido en una realidad que está transformando la cultura política de los responsables políticos y, por consiguiente, su forma de ejercer la política. Por lo tanto, la diputación tiene vías y oportunidades para su propia transformación”) sino también social y cultural ("Etorkizuna Eraikiz tiene un gran potencial transformador del territorio").

En definitiva, lo que los participantes reflejan es una mayor conciencia de haber estrechado relaciones en el seno del equipo de gobierno y con ello haber crecido en confianza para llevar a cabo cambios organizacionales, como también para atribuir a su organización una mayor capacidad transformadora en un nivel más amplio, el de la sociedad a la que se quieren involucrar. De manera colaborativa, los participantes han explicitado entonces la potencialidad de las relaciones grupales así como de las relaciones con la organización y con la sociedad; esto respalda la idea extraída de la revisión de la literatura sobre capital social de que las relaciones sociales benefician no solo a los individuos sino también a las organizaciones, llevándolas más allá de sí mismas, para llegar a hacer cosas que sin la colaboración no hubieran sido posibles y, en último término, para establecer comunidades vivas y promover el desarrollo. Tras el proceso de aprendizaje el equipo de gobierno se siente más capaz de llevar a cabo un proyecto de transformación social que implica a la sociedad. 


\section{Aspecto 5: Aprendizaje sobre la escucha a la sociedad: Incrementando el deseo de compartir la autoridad}

Por lo que respecta a lo aprendido en torno a la escucha a la sociedad, los datos muestran una evolución que va de una constatación de disparidad de criterios dentro del grupo sobre lo que la escucha es e implica, a una mayor capacidad colectiva para implicarse en la cocreación con la sociedad. Los datos se recogen en el cuadro 6.

CUADRO 6. Aspecto 5: Evolución del aprendizaje sobre la escucha a la sociedad ¿Qué has aprendido hoy sobre la escucha a la sociedad?

$\begin{array}{ll}\text { Sesión AL 1 } & \text { Que hay disparidad en el grupo sobre qué es la sociedad y qué significa escucharla. } \\ \text { Sesión AL 2 } & \begin{array}{l}\text { La necesidad de establecer mecanismos para estar cerca de la sociedad y escucharla } \\ \text { activamente. }\end{array} \\ \text { Sesión AL 3 } & \begin{array}{l}\text { Que al conocer más a la sociedad, se genera mayor confianza para ejercer una gobernanza } \\ \text { colaborativa. }\end{array} \\ \text { Sesión AL 4 } & \text { Que hay deseo de apostar por la cocreación para ejercer el liderazgo compartido. }\end{array}$

Fuente: Elaboración propia.

Los datos reflejan que, al comienzo del proceso de aprendizaje, la sociedad significa algo diferente para cada responsable político y, por lo tanto, la relación con esta también se entiende de forma diversa. Se constata además desconocimiento e incertidumbre sobre los cambios que se están produciendo en la sociedad actual y sobre cómo conectar con esta. En palabras de los participantes "es fundamental establecer la definición clara de lo que significa escuchar a la sociedad para llevarla a la práctica y lograr conectar".

Es recurrente en las respuestas el escepticismo sobre la capacidad de deliberación de la sociedad para hacer frente a este tipo de problemas. Pero este escepticismo no es incompatible con el compromiso de los líderes por estar cerca de ella y escucharla activamente: "Necesitamos definir qué estamos dispuestos a cambiar o a incorporar en nuestra forma de trabajar como resultado de esa 'nueva' escucha'. Varias respuestas muestran el deseo de pasar de una posición en la que lo que se persigue es que la ciudadanía escuche a otra en la que realmente se le quiera escuchar. 
CUADRO 7. La aportación del aprendizaje de escucha al capital social

Características del capital social y relación con la escucha

Surge de las conexiones entre los individuos, las redes sociales y las normas de reciprocidad y confianza (Putnam, 2000).

Se apoya en el valor que aportan las relaciones a la persona y organización individual, por cuanto las relaciones son fuente de desarrollo (Coleman, 1990; Lin, 2001; Portes, 1998; Putnam, 1993; Barreiro, 2000; Gallicchio, 2004).

Facilita la colaboración y el compromiso, el flujo de información, e incrementa el sentido de pertenencia y de obligación social (Saffer, 2016).

Es un recurso intangible de las organizaciones, que permite acceder a bienes y recursos tangibles que de otra forma serían inalcanzables para los individuos y las organizaciones (Canel y Luoma-aho, 2019; Coleman, 1988; Bourdieu, 1983).

Parte del valor intangible que genera se puede medir en términos de confianza y confiabilidad (Coleman, 1990; Fukuyama, 1995).

Se construye mediante procesos de interacción social en los que se comparten las definiciones de los problemas y de las soluciones (Saffer, 2016).

La escucha es clave para generar las relaciones que están en la base del capital social (Hazelton y Kennan, 2000; Luoma-aho, 2013; Canel y Luoma-aho, 2019).

Los procesos de capital social parten de un estrechamiento de relaciones en niveles micro, para escalar a niveles macro haciendo redundar la cercanía y confianza a grupos y sociedades más extensas (Putnam et al., 1993; Putnam et al., 2003).

Como es relacional e intangible, para generarlo hacen falta procesos de largo plazo. Resulta de la acumulación de experiencias positivas que tienen los implicados en un intercambio de comunicación y escucha, así como de la explicitación de tales experiencias (Canel y Luoma-aho, 2019).
Resultados del aprendizaje de los líderes públicos

Sobre el aprendizaje: Cuando se crean las condiciones para la escucha en un grupo, se estrechan unas relaciones con las que se puede hacer explícito el valor latente en las mismas.

Sobrela persona individual: El aprendizaje de escucha crea espacios compartidos en los que se puede pasar de definiciones individuales a visiones comunes de los problemas, las soluciones y los retos de futuro.

Sobre los problemas de la gestión pública: Los espacios compartidos ayudan a explicitar conexiones entre el nivel micro (el equipo de gobierno) y el macro (las organizaciones sociales y los ciudadanos).

Sobre la organización y el modelo de gobernanza colaborativa: La explicitación de conexiones entre lo micro y lo macro refuerza el reconocimiento de la potencialidad de transformación social del proyecto gubernamental.

Sobre la escucha a la sociedad: Al explicitar lo aprendido sobre la escucha, se incrementa el deseo de compartir la autoridad con los ciudadanos, y se refuerza la capacidad y deseo de apostar por la coparticipación.

Fuente: Elaboración propia. 
A medida que se avanza en el proceso de aprendizaje, se pone de manifiesto una mayor confianza por parte de los líderes públicos en la propia ciudadanía para ejercer la gobernanza compartida. Reveladora es, en este sentido, la siguiente respuesta de un participante: "La sociedad está dispuesta a escuchar y a participar. ¿Estamos nosotros como líderes y como organización, dispuestos a compartir el liderazgo y, por tanto, la decisión?" El proceso ha puesto a prueba la autenticidad del deseo de los líderes públicos de otorgar autoridad a la sociedad.

Al finalizar el proceso, parece consolidarse en el grupo el deseo de cocrear, de aprender a hacerlo, de desarrollar mecanismos de escucha para sistematizar no solo la recolección de la opinión de la sociedad, sino para asegurar que esta opinión queda finalmente reflejada en la toma de decisiones. En este sentido son reveladoras las afirmaciones de dos participantes en la última sesión: "Hoy me siento más capaz de actuar con el grupo y con la sociedad"; "queremos que la escucha se refleje en las políticas públicas".

Parece que se puede afirmar a partir de estos resultados que la explicitación del aprendizaje de escucha ha hecho también explícita la existencia de unas relaciones que aportan valor tanto al grupo como a la sociedad y la predisposición de los líderes de otorgar autoridad a esa red de relación más extensa; y se han revelado así formas de trabajar en la gestión pública que pueden ser beneficiosas para el conjunto de los ciudadanos. El cuadro 7 sintetiza la discusión de los resultados.

\section{CONCLUSIÓN}

Esta investigación ha explorado las relaciones entre la escucha y el capital social con el fin de identificar políticas gubernamentales con las que se pueda aspirar a potenciar este recurso intangible relacional y fortalecer así a la sociedad. Lo ha hecho mediante una reflexión del proceso de aprendizaje de escucha emprendido por un equipo de gobierno con el fin de desarrollar la gobernanza colaborativa.

¿Qué aporta al capital social la reflexión colaborativa de líderes públicos sobre su aprendizaje de escucha? La investigación muestra que cuando líderes públicos deciden aprender a escuchar conjuntamente, explicitan los valores latentes en las relaciones que establecen y, con ello, se predisponen a identificar y articular el capital social, y a trabajar más a favor del mismo. Esto es así porque, siguiendo los distintos niveles de observación del proceso de aprendizaje de esta investigación, se ha puesto de manifiesto que: a) cuando un equipo de gobierno quiere aprender a escuchar conjuntamente, estrecha las relaciones con sus pares, explicitando el valor latente que reside en las mismas; $b$ ) al hacerlo, se pone en juego una interacción que crea un espacio compartido en el que se llegan a definiciones comunes de 
los problemas, las soluciones y los retos de futuro; c) se explicitan entonces conexiones entre un nivel micro (el gobierno) y el macro (la sociedad), incrementándose la conciencia de que lo que se alcanza socialmente tiene más valor que lo que tendría la suma de la resolución individual de los problemas individuales; d) los líderes públicos se sienten entonces más capaces de avanzar en la gobernanza colaborativa al incrementar su confianza en el alcance e impacto transformacional que tiene su gestión pública; e) como consecuencia, se refuerza el deseo de los líderes públicos de otorgar autoridad a la sociedad a la que quieren implicar y, con ello, de extender la red de relaciones que se haría "propietaria" de la toma de decisiones de la gestión pública. En definitiva, el proceso de aprendizaje ha revelado a los miembros de un gobierno formas de trabajar con las cuales se puede desarrollar capital social y, en consecuenica, beneficiar a la sociedad.

Bien es verdad que esta investigación no ha computado el capital social generado - entre otras cosas porque, tal y como se ha argumentado, este recurso intangible deriva de procesos de largo plazo-; cuestión, por lo tanto, para la que habrá que diseñar una investigación ad hoc cuando haya suficiente perspectiva temporal. Tampoco ha evidenciado si hubo o no escucha real a la sociedad. Su objetivo era explorar un proceso de aprendizaje con el fin de identificar si intervenciones como la llevada a cabo pueden ser de utilidad para los programas gubernamentales de gobernanza colaborativa. Por eso, lo que esta investigación permite afirmar es que el aprendizaje de la escucha por parte de líderes públicos ayuda a crear las condiciones — una mayor predisposiciónpara el establecimiento del tipo de relaciones que están en la base de la generación del capital social.

La extrapolación de estos resultados a otras regiones o gobiernos debe tener en cuenta, también, otras limitaciones. Primero, que el terriotorio observado goza, como se ha mencionado, de un nutrido tejido social, y si se quisiera replicar este proceso de aprendizaje habría que identificar si antes son necesarias otras estrategias para generar motivación. En el contexto español, existen pocos estudios científicos que analicen y radiografíen el panorama de la escucha y la comunicación en las diputaciones provinciales y forales en su conjunto (véase por ejemplo Fernández-Souto et al., 2019), y sería de utilidad llevar a cabo más trabajos que abarcaran los diferentes contextos. Segundo, que la muestra se ha ceñido al equipo de gobierno, que es quien se implicó en el proceso de aprendizaje, por lo que será interesante ver qué ocurre con intervenciones que abarquen no solo a las personas con función ejecutiva de gobierno, sino también a personal técnico, así como a representantes de la sociedad. 
A la luz de esta investigación se pueden realizar las siguientes consideraciones sobre las implicaciones operativas para la gestión pública.

Primero, los resultados proporcionan varias ideas para implementar procesos de aprendizaje activo aplicados a la gestión pública. La investigación ha puesto de manifiesto los beneficios de acompañar los programas gubernamentales participativos de un proceso de aprendizaje con metodología de action learning. La gobernanza colaborativa requiere del involucramiento de los líderes públicos en la reflexión-aprendizaje-acción como parte del proceso de escucha y aprendizaje social. Se hace recomendable trabajar para instaurar esta dinámica al interior de las organizaciones públicas para establecer mecanismos de colaboración y compromiso entre quienes ejercen la toma de decisiones y la sociedad.

Segundo, de la experiencia analizada se desprende que, en la gestión pública hay que confiar más en el impacto que pueden tener las acciones que se llevan a cabo en un nivel micro. Los programas gubernamentales de compromiso ciudadano y participación social tienen el peligro de caer en maximalismos fruto de la presión por lograr un amplio alcance. El capital social que, bien es verdad, no deja de ser un constructo, permite analizar el funcionamiento de las sociedades; $y$ en este caso ha dado a conocer que un estrechamiento de relaciones al interior de un equipo de gobierno puede ser un buen comienzo de programas que quieran llegar a implicar a toda la sociedad.

Tercero, y último, trabajar a favor del desarrollo de un territorio requiere programas que se enfoquen en los recursos intangibles. Aun cuando esta investigación no haya recopilado evidencias sobre lo tangible, permite afirmar que el capital intangible da acceso al tangible en el sentido de que el capital social está asociado a una cohesión gracias a la cual se puede ser más eficaz y eficiente. El capital social no va simplemente de estar bien juntos, de desarrollar relaciones satisfactorias; es, más bien, extraer valor — capital— de unas relaciones que, gracias a que existen, se pueden llevar a cabo acciones que tienen más valor que si se actuara por separado. Queda por ver en futuras investigaciones si del proceso observado surgen, efectivamente, políticas públicas cuya "propiedad" es compartida.

A nuestro entender, esta conclusión adquiere especial valor en el contexto de la crisis ocasionada por el covid-19: la superación de la misma se está librando en la capacidad de la ciudadanía de verse a sí misma como parte de la solución, para lo que está siendo nuclear el sentimiento de confianza de que solo juntos se puede combatir la pandemia. En este sentido serán interesantes las investigaciones que se puedan desarrollar para analizar, por ejemplo, el \#EsteVirusloParamosEntreTodos. La respuesta al covid-19 descansa no sólo en la articulación del trabajo 
de las autoridades públicas, como tampoco solo en el del personal sanitario, de las fuerzas de seguridad, de los transportistas o de los medios de comunicación. Se trata de una crisis para la que resulta clave la convicción de cada ciudadano de que lo poco que tiene en sus manos (confinarse en su casa) adquiere gran valor cuando se suma al conjunto de lo que hace el resto de la comunidad. Es, en definitiva, la convicción de que se llega más lejos cuando se aúnan energías. A la luz de esta investigación es deseable que a futuro se desarrollen investigaciones y programas gubernamentales para responder con más capital social a crisis como la ocasionada por la pandemia del coronavirus. $\mathbf{G} \cong \tilde{\Xi}$

\section{REFERENCIAS}

Åberg, M. y M. Sandberg (2017), Social Capital and Democratisation: Roots of Trust in PostCommunist Poland and Ukraine, Abingdon, Routledge.

Barreiro, F. (2000), "Desarrollo desde el territorio: A propósito del desarrollo local", Chile: Red de Desarrollo Económico Local, pp. 1-28.

Bellah, R., R. Madsen, W. Sullivan, A. Swidler y S. Tipton (1991), The Good Society, Nueva York, Knopf.

Beshi, T.D. y R. Kaur (2019), "Public Trust in Local Government: Explaining the Role of Good Governance Practices”, Public Organization Review, 20, pp. 337-350.

Bolívar, G. y L. Flores (2011), "Discutir el campo del capital social desde un enfoque transdisciplinario", Polis: Revista Latinoamericana, 10(29), pp. 55-80.

Borner, M. y A. Zerfass (2018), "The Power of Listening in Corporate Communications: Theoretical Foundations of Corporate Listening as a Strategic Mode of Communication", en S. Bowman, A. Crookes, Ø. Ihlen y S. Romenti (eds.), Public Relations and the Power of Creativity: Strategic Opportunities, Innovation and Critical Challenges, Bingley, Emerald Publishing Limited, pp. 3-22.

Bourdieu, P. (1983), “The Forms of Capital”, en J. Richardson (ed.), Handbook of Theory and Research for the Sociology of Education, Nueva York, Greenwood Press, pp. 241-258.

Canel, M.J. (2018), La comunicación de la administración pública: Para gobernar con la sociedad, Ciudad de México, Fondo de Cultura Económica.

Canel, M.J. y V. Luoma-aho (2019), Public Sector Communication: Closing Gaps between Citizens and Organizations, Boston, Wiley-Blackwell.

Canel, M.J., E. Oliveira y V. Luoma-aho (2017), "Exploring Citizens' Judgments about the Legitimacy of Public Policies on Refugees: In Search of Clues for Governments' Communication and Public Diplomacy Strategies", Journal of Communication Management, 21(4), pp. 355-369.

Carvalho, A., Z. Pinto-Coelho y E. Seixas (2019), "Listening to the Public-Enacting Power: 
Citizen Access, Standing and Influence in Public Participation Discourses", Journal of Environmental Policy \& Planning, 21(5), pp. 563-576.

Coleman, J. (1988), "Social Capital in the Creation of Human Capital”, American Journal of Sociology, 94, pp. S95-S120.

Coleman, J. (1990), Foundations of Social Theory, Cambridge, Belknap Press of Harvard University Press.

Crosby, B. y J. Bryson (2017), "Why Leadership of Public Leadership Research Matters: And What to Do about It", Public Management Review, 20(9), pp. 1265-1286.

Diputación Foral de Gipuzkoa (2019), Modelo de gobernanza Etorkizuna Eraikiz, San Sebastián, Diputación Foral de Gipuzkoa.

Dobson, A. (2012), "Listening: The New Democratic Deficit", Political Studies, 60(4), pp. 843-859.

Dodd, M., J. Brummette y V. Hazleton (2015), "A Social Capital Approach: An Examination of Putnam's Civic Engagement and Public Relations Roles", Public Relations Review, 41(4), pp. 472-479.

Ercan, S.A., C. Hendriks y J. Dryzek (2019), "Public Deliberation in an Era of Communicative Plenty”, Policy \& Politics, 47(1), pp. 19-36.

Fernández, M., N. Khalil y M. del Pilar Gómez (2018), "La escucha organizacional como estrategia de comunicación: Análisis de su gestión en España”, Revista Internacional de Relaciones Públicas, 8(15), pp. 197-214.

Fernández-Souto, A.B., I. Puentes-Rivera y M. Vázquez-Gestal (2019), “Gestión y profesionalización de las relaciones públicas en las diputaciones provinciales y forales", Gestión y Política Pública, XXVIII(2), pp. 541-568.

Forester, J. (1989), "Planning in The Face of Power", Journal of the American Planning Association, pp. 27-47.

Fukuyama, F. (1995), Trust: The Social Virtues and the Creation of Prosperity, Nueva York: Free Press.

Gallicchio, E. (2004), "El desarrollo local: ¿Cómo combinar gobernabilidad, desarrollo económico y capital social en el territorio?”, Cuadernos del CLAEH, 27(89).

Goodin, R. (2008), Innovating democracy: Democratic Theory and Practice after the Deliberative Turn, Nueva York, Oxford University Press.

Gutiérrez, A. (2008), "Redes e intercambio de capitales en condiciones de pobreza: Dimensión relacional y dimensión vincular", Redes, 14(1), pp. 1-17.

Hazelton, V. y W. Kennan (2000), "Social Capital: Reconceptualizing the Bottom Line", Corporate Communications: An International Journal, 5(2), pp. 81-86.

Heifetz, R. y M. Linsky (2017), Leadership on the Line, with a New Preface: Staying Alive through the Dangers of Change, Brighton: Harvard Business Press. 
Heifetz, R. y M. Linsky (2017), Leadership on the Line, with a New Preface: Staying Alive through the Dangers of Change, Brighton, Harvard Business Press.

Hyyppä, M. y J. Mäki (2003), "Social Participation and Health in a Community Rich in Stock of Social Capital”, Health Education Research, 18(6), pp. 770-779.

Johnson, A. (2007), Speech Putting People First, disponible en: http://www.dh.gov.uk/en/ News/Speeches/DH_081194 [fecha de consulta: 20 de noviembre de 2019].

Johnston, K.A. y A. Lane (2018), "Building Relational Capital: The Contribution of Episodic and Relational Community Engagement”, Public Relation Review, 44(5), pp. 633-644.

Korten, D.C. (1981), “The Management of Social Transformation”, Public Administration Review, 41(6), pp. 609-618.

Lin, N. (2001), Social Capital: A Theory of Social Structure and Action, Cambridge, Cambridge University Press.

Luoma-aho, V. (2013), "Corporate Reputation and the Theory of Social Capital”, en C. Carroll (ed.), The Handbook of Communication and Corporate Reputation, Chichester, Wiley-Blackwell, pp. 279-290.

Luoma-aho, V., M. Vos, R. Lappalainen, A.M. Lämsä, O. Uusitalo, P. Maaranen y A. Koski (2012), "Added Value of Intangibles for Organizational Innovation", Human Technology: An Interdisciplinary Journal on Humans in ICT Environments, 8(1), pp. 7-23.

Macnamara, J. (2015), Creating an "Architecture of Listening" in Organizations: The Basis of Engagement, Trust, Healthy Democracy, Social Equity, and Business Sustainability, Sidney, University of Technology Sydney.

Macnamara, J. (2016), "Organizational Listening: Addressing a Major Gap in Public Relations Theory and Practice”, Journal of Public Relations Research, 28(3-4), pp. 146-169.

Macnamara, J. (2017), “Toward a Theory and Practice of Organizational Listening”, International Journal of Listening, 32(1), pp. 1-23.

Murphy, A., M.J. Canel y X. Barandiarán (2020), "How do Public Leaders Learn from Society? A reflexive Analysis of Action Learners", Action Learning: Research and Practice, 17(2), pp. 172-185. ISSN 14767341, 14767333.

Needham, C. (2001), Listen and Lead-the Challenge from the Women of Worcester, Londres, OpenDemocracy.

Ordóñez, G. y W. Ruiz Ochoa (2015), “Formación de capital social comunitario a partir de programas orientados a combatir la pobreza en México: El impacto de Hábitat”, Gestión y Politica Pública, XXIV(1), pp. 3-49.

Ospina, S. (2016), "Collective Leadership and Context in Public Administration: Bridging Public Leadership Research and Leadership Studies”, Public Administration Review, 77(2), pp. 275-287.

Pedler, M. (2011), Action Learning in Practice, Brookfield, Gower Publishing. 
Piqueiras, P., M.J. Canel y V. Luoma-aho (2020), "Citizen Engagement and Public Sector Communication”, en V. Luoma-aho y M.J. Canel (eds.), Handbook of Public Sector Communication, Chichester, Wiley-Blackwell, pp. 277-288.

Plati, C. (2005), "Listen”, Social Policy, 35(3), pp. 49-50.

Portes, A. (1998), "Social Capital: Its Origins and Applications in Modern Sociology", Annual Review of Sociology, 24(1), pp. 1-24.

Prieto, R. y R. Fabelo (2009), "Liderazgo y capital social: Uso de redes como herramienta para el desarrollo sostenible”, Telos, 11(1), pp. 52-68.

Putnam, R. (1993), "The Prosperous Community”, The American Prospect, 4(13), pp. 35-42.

Putnam, R. (2000), Bowling Alone: The Collapse and Revival of American Community, Londres, Simon \& Schuster.

Putnam, R. (2010), "Para superar conflictos hay que escucharse entre diferentes", El Diario Vasco, 15 de septiembre, disponible en: https://www.diariovasco.com/v/20100915/ politica/para-superar-conflictos-escucharse-20100915.html [fecha de consulta: 29 de abril de 2020].

Putnam, R.D., R. Leonardi y R. Nanetti (1993), Making Democracy Work: Civic Traditions in Modern Italy, Princeton, Princeton University Press.

Putnam, R., L. Feldstein y D. Cohen (2003), Better Together: Restoring the American Community, Nueva York, Simon \& Schuster.

Ramírez de la Cruz, E.E. (2019), "Reseña de 'La comunicación de la administración pública. Para gobernar con la sociedad”, Gestión y Politica Pública, XXVIII(1), pp. 279-282.

Revans, R.W. (1998), "Sketches in Action Learning”, Performance Improvement Quarterly, 11(1), pp. 23-27.

Revans, R. (2011), ABC of Action Learning, Farnham, Gower.

Richards, J. (2008), Rhetoric: The New Critical Idiom, Londres, Routledge.

Saffer, A. (2016), "A Message-focused Measurement of the Communication Dimension of Social Capital: Revealing Shared Meaning in a Network of Relationships", Journal of Public Relations Research, 28(3-4), pp. 170-192.

Simmons, R. (2011), "Leadership and Listening: The Reception of User Voice in Today's Public Services", Social Policy \& Administration, 45(5), pp. 539-568.

Southard, B. y A. Wolvin (2009), “Jimmy Carter: A Case Study in Listening Leadership", The International Journal of Listening, 23(2), pp. 141-152.

Stivers, C. (1994), “The Listening Bureaucrat: Responsiveness in Public Administration”, Public Administration Review, 54(4), pp. 364-369.

Suárez Rincón, J.V., Y.V. Aranda Camacho y F. Herrera-Tapia (2018), "Las redes de conocimiento para la gestión territorial: El caso de la Red GTD”, Gestión y Politica Pública, XXVII(2), pp. 541-570. 
Whiteley, P., H. Clarke y D. Sanders (2016), "Why do Voters Lose Trust in Governments? Public Perceptions of Government Honesty and Trustworthiness in Britain 2000-2013”, The British Journal of Politics and International Relations, 18(1), pp. 234-254.

Wilson, P. (1997), "Building Social Capital: A Learning Agenda for the Twenty-first Century", Urban Studies, 34(5-6), pp. 745-760.

Yang, A. y M. Taylor (2013), "The Relationship between the Professionalization of Public Relations, Societal Social Capital and Democracy: Evidence from a Cross-national Study”, Public Relations Review, 39(4), pp. 257-270. 
ANEXO I. Características de los participantes en el proceso de action learning (equipo de gobierno provincial)

Nivel jerárquico / área que participa

Tipo de participante

Diputado general de Gipuzkoa

Diputado de Gobernanza y Relación con la Sociedad

Diputado de Hacienda

Diputada de Política Social

Diputada de Infraestructuras viarias

Diputado de Promoción Económica y Desarrollo Rural

Responsable de Gabinete del Diputado General

Directora general de Comunicación del Gabinete del Diputado General Asesor de Comunicación del Diputado General

Asesor de Comunicación del Diputado General

Director de Estrategia del Gabinete del Diputado General

Jefe de Servicio de la Dirección de Estrategia
Funcionario electo

Funcionario de designación política

Funcionario de designación política

Funcionario de designación política

Funcionario de designación política

Funcionario de designación política

Funcionario de designación política

Funcionario de designación política

Funcionario de designación política

Funcionario de designación política

Funcionario público de carrera

Funcionario público de carrera

Fuente: Elaboración propia. 
Xabier Barandiaran es doctor en Sociología y profesor titular de la Facultad de Ciencias Sociales y Humanas de la Universidad de Deusto, donde imparte clases en el campus de Donostia-San Sebastián. Sus publicaciones se centran principalmente en la comunicación estratégica y el capital social. En los últimos años ha estado trabajando en temas relacionados con la cultura política y el capital social. Forma parte del equipo de investigación en comunicación. Otras áreas de interés de investigación incluyen los valores y la cultura política.

Anne Murphy tiene un MA en Gestión del Aprendizaje por Lancaster University, universidad en la que actualmente cursa sus estudios de doctorado. Estudia etnografía lingüística del liderazgo y del habla laboral, explorando la interacción entre los profesionales para llevar a cabo cambios en las ideas y las prácticas. Especializada en el aprendizaje organizacional, cuenta con 30 ańos de experiencia en la investigación aplicada y participativa en las organizaciones. Ha trabajado en diferentes sectores con personas procedentes de contextos profesionales diversos. En su carrera como consultora ha trabajado internacionalmente con clientes tanto públicos como privados y del tercer sector, utilizando metodologías como la action research y el action learning para desarrollar iniciativas de cambio colaborativo en diferentes situaciones: fusiones, adquisiciones, alianzas, gestión del conocimiento, trabajo multi-agente, desarrollo comunitario, liderazgo de gente joven, desarrollo de la cooperación, e innovación sanitaria. Desde 2002 se ha concentrado en programas de investigación organizacional así como aprendizaje aplicado en facultades de administración pública, gestión, y medicina de importantes universidades europeas.

María José Canel es catedrática de Comunicación Política y Sector Público, Universidad Complutense Madrid (España). Co-Chair Public Marketing and Communication, European Group of Public Administration (EGPA). Presidenta fundadora de Asociación de Comunicación Política (ACOP). Autora líder, es Victory Awards (Washington, DC, 2016). Con más de un centenar de publicaciones sobre comunicación de gobiernos y Administración Pública e intangibles en sector público. Entre sus títulos están: Comunicación de la Administración Pública, Public Sector Communication, Government Communication, Political Scandals, Comunicación Política. Ha publicado en España, EUA, Reino Unido, Italia, Polonia, Brasil, México, Colombia, Argentina. Tiene experiencia práctica: fue Directora General del gabinete de un ministerio, ha trabajado en campañas electorales y en la comunicación de gobiernos de diferentes países; y en el training de comunicadores públicos (en España, Francia, Holanda, México, Colombia, Guatemala, Argentina y Chile). 